\section{Current affairs}

\section{Peter D. Killworth}

General Circulation of the Ocean. Edited by Henry D. I. Abarbanel and W. R. Young. Springer-Verlag: 1987. Pp.291. DM 160.

THERE are probably three main classes of books on science. Textbooks, with a readership of students and researchers; simplified accounts of developments in a field, intended for the intelligent layman or the scientist who wants to broaden his knowledge; and books that assume some understanding of the subject by the reader, and aim to convey the beliefs, rationales and many of the methods of active researchers. A book in this third class is a rare phenomenon. Unless it is written as a monograph by one individual, it requires a lot of coordination by its editors: one man's trivia can be another man's important material. To succeed in the marketplace, such a book must "feel" different from those in the other two classes.

General Circulation of the Ocean lies in this third category. Its aim is to pass on the current thinking about the behaviour of the ocean on long time scales, using the contents of five sets of lectures given by the authors in 1983. The selection of material achieves that aim well. It also, remarkably, gives a clear impression of the authors delivering their lectures at a blackboard, with asides like "I'll come clean at the outset and state my conclusion"; thus the "feel" is there. Some readers may be irritated by this style; I enjoyed it. However, those present at the lectures were given the opportunity to ask questions - the book's reader cannot. There will therefore be readers who wonder what the terms in the table on p. 22 conveyed in the paper cited, or who seek in vain for ' $(4.25 \mathrm{a})$ ' on p. 173 ; or who wonder where Young's equation (3.13), which conflicts with Hendershott's (paragraph 1.6.9), came from (Hendershott's is the correct one.) These are symptoms of the many minor lapses in editing, which together with the atrocious English ("plateauing", "dimensionfull") and poor diagram quality - most are apparently photocopies from papers - leave one unconvinced that enough care went into the book's production. Whether this matters depends on the readership. Experts, dipping into the book, can fill in the gaps without difficulty; students would have more trouble.

I would have liked Niiler's admirable distillation of observations related to theory to have been twice the length; however, the organizers insisted that "the number of lectures be moderately finite"! Pedlosky's survey of classical (1970s) thermocline theory, plus 1980s updates of layered "ventilated" theory, shows one of the many excellent features of the book: the quality of total honesty with the reader. There is a temptation when writing a paper to conceal under a plethora of algebra the less successful elements of one's model. That is not so here: strong and weak points are discussed together.

Veronis's section on inverse modelling (the attempt to find a "best fit" velocity field given observations of temperature and salinity data) is the shortest in the book. However, in terms of the amount of immediately useful information and advice for the researcher, it weighs in as the heaviest, and is, simply, the best description of inverse methodology I have read. Young's lectures on baroclinic theories of wind driven circulation overlap somewhat with Hendershott's more historical review of barotropic circulation and some pruning could usefully have been done here. Both are good treatments, although the 20 pages allotted to eddy flux parameterisations seems over long for the end results. Unlike the other lectures, both sometimes let pages of mathematics pass by without the occasional physical discussion, which would have been useful.

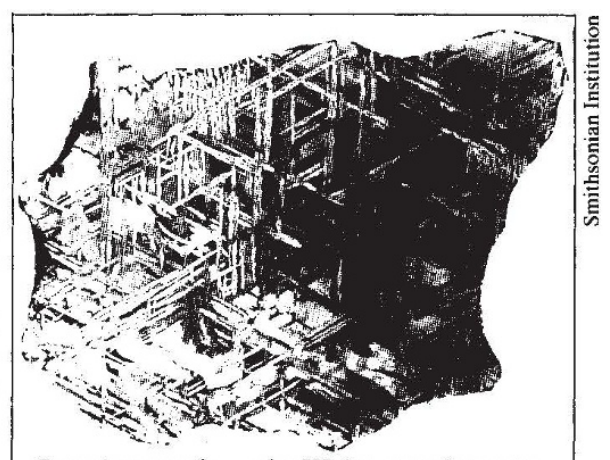

Cosmic crystals - the Waingaromia meteorite from New Zealand, showing the Widmanstatten pattern formed by intergrowth of kamacite plates within a taenite lattice. The picture is taken from Meteorites and Their Parent Planets, by Harry $Y$. McSween Jr, newly published by Cambridge University Press. Price is $£ 15, \$ 24.95$.

But the most important feature of the book is that it fills the reader with a sense of excitement. Ocean circulation studies are exciting and this book lets the reader know it.

Peter D. Killworth is a Principal Scientific Officer at the Deacon Laboratory, Institute of Oceanographic Sciences, Wormley, Godalming, Surrey GU8 $5 U B, U K$.

\section{Solid contribution}

\section{Colin Bates}

Crystalline Semiconducting Materials and Devices. Edited by Paul N. Butcher, Norman H. March and Mario P. Tosi. Plenum: 1986. Pp.645. \$107.40.

ONE of the most popular lines of solid state research in both physics and chemistry is concerned with various types of semiconductors. Apart from their intrinsic scientific interest, semiconductors have many widespread applications which enter into several areas of high technology. New materials are continuously being sought and the literature is full of new results and ideas. Interest originally centred on the bulk materials but more recently the emphasis has moved to the study of ultra thin films and the interface between two materials and superlattices. Another aspect of current research is the careful control of impurities, imperfections and dopants intentionally added during the manufacture.

Such a wide-ranging activity needs some comprehensive base from which adequate but simple models can be built, thus providing the necessary framework upon which the properties of the various materials can be characterized and compared. This book serves admirably the requirement of such a base. It is made up of 16 independently written chapters from 17 contributions from well-known
European and Japanese universities and laboratories. It begins by discussing the structure of bonds and bands in perfect semiconductors and then goes on to consider thermal and electrical conduction in crystalline structures, followed by a discussion of the effects on the band structure achieved by including shallow and deep impurities centres. After this examination of fundamental work on bulk materials, surface and interface effects are discussed. The last four chapters are concerned with semiconductors as devices. For example, their use in optical communication is discussed in some detail.

In my opinion, this book has succeeded in its attempt to provide a complete account of the basic physics and chemistry of semiconductors. The chapters have been carefully written, although, as is usual in a book of this type, cross-referencing between the chapters is not very frequent. The earlier chapters, being much more fundamental, rely on the more traditional and standard aspects of the basic theory. In contrast, the chapters dealing with the application to devices contain far more references to recent work - up to 1984.

Both graduate research students and more experienced research workers should find the book an invaluable reference source to enable them to understand the more advanced and specialized research paper.

Colin Bates is a Professor in the Department of Physics, University of Nottingham, Nottingham $N G 72 R D, U K$. 